\title{
L'AVENIR DE LA RADIODIFFUSION INTERNATIONALE
}

\author{
Bernard Wuilleme ${ }^{1}$
}

La question de l'avenir de la radiodiffusion internationale, pour ne pas dire de sa survie, cette question est posée depuis la Chute du Mur de Berlin le 9 novembre 1989, même si cette date est symbolique.

En effet, depuis sa naissance depuis les années 1930, la radiodiffusion internationale n'a cessé d'avoir un rôle paradoxal.

Si sa création a des motifs bien précis : informer ses ressortissants à l'étranger de ce qui se passe en métropole, la réalité deviendra rapidement très différente pour devenir un outil de propagande aux mains des Etats. Car faut-il le rappeler : la radiodiffusion internationale est une radio financée, dirigée (ses directeurs sont nommés par le gouvernement du pays) par les États.

Bref rappel de cette « histoire de la radiodiffusion internationale » :

- 1930-1939 : Période d'avant-guerre où les forces de l'Axe s'intéressent aux publics récepteurs des pays colonisés ou sous mandat pour les convaincre de se rebeller contre la métropole. Bien entendu contre-propagande des États colonisateurs sur les mêmes publics.

1 Professeur en Sciences de l'Information et de la Communication Université Jean Moulin Lyon 3

Recherches en communication, $\mathrm{n}^{\circ} 26$ (2006). 
- 1939-1945 : Véritable «guerre des ondes » entre d'une part les Alliés (Grande-Bretagne, France, États-Unis, Hollande, Belgique, etc) qui contre carrent les actions de propagande, de désinformation, d'intoxication des forces ennemies (Allemagne, Italie, Japon).

- 1945-1989 : La radiodiffusion internationale va vivre sa plus grande période pendant la Guerre froide. Une confrontation bi-polaire Est-Ouest, avec d'un côté la Russie et les Etats qui se trouvent derrière le rideau de fer auxquels se rajoutent l'Albanie, la Chine. De nouveaux interlocuteurs aussi bien à l'Ouest : RFE et RL et à l'Est avec Radio et Progrès.

À la fin de cette période s'est posée la question de l'utilité et donc l'avenir de la radiodiffusion internationale. À quoi pouvait bien servir une radio de ce type ? En effet, le monde n'était plus bi-polaire, mais devenait multipolaire, la raison d'être de communiquer avec les colonies n'avaient plus cours puisque tous les pays colonisés étaient devenus indépendants autour des années 1960. Bref, où étaient les amis, où étaient les ennemis?

Comme personne ne peut prédire l'avenir, nous exposerons notre point de vue selon 3 approches :

- Nous ferons des constats sur ce qui s'est produit depuis 1989 selon 2 approches : techniques et radiophoniques internationaux.

- Nous formulerons quelques hypothèses ;

- Nous tenterons d'en dégager une synthèse sous forme d'un scénario possible.

\section{Les constats}

La chute du Mur de Berlin a eu pour conséquence de modifier complètement le paysage de la radiodiffusion internationale. En effet, sans reprendre la chronologie des faits, il faut retenir qu'à partir du moment où l'URSS est morte (1990), tout change derrière le rideau de fer. Chaque État retrouvant son indépendance politique et radiophonique.

De même qu'à l'Ouest, il faudra reconvertir ces radios de propagande en radios «classiques » à vocation internationale.

Que constatons-nous? 


\section{Sur le plan technique}

Apparition des nouvelles technologies :

- d'émission : internet, satellite (programme World Space), numérique (radio numérique, compression numérique).

Les ondes courtes qui sont « capricieuses » et surtout coûteuses commencent à être abandonnées par certains radiodiffuseurs. La Suisse sera le premier pays à l'annoncer et d'autres suivent et suivront.

- de réception : DRM, DAB, radio à dynamo (qui ne nécessite aucune alimentation électrique), podcast.

\section{Sur le plan radiophonique international}

Les pays sous la tutelle de l'Union soviétique mais ne faisant pas partie de l'Union soviétique et qui tranchent rapidement avec les anciens gouvernants (Hongrie, Pologne, Tchécoslovaquie) retrouvent très rapidement un service extérieur de radiodiffusion et mettent en place un système semblable aux pays de l'Ouest.

Les pays de l'Est qui ne sont pas parvenus à rompre rapidement avec les anciens gouvernants seront plus lents à mettre en place un service extérieur, comme la Bulgarie et la Roumanie.

Les pays qui étaient sortis du giron soviétique comme la Yougoslavie et l'Albanie subissent une remise en question radicale sur les plans politiques et économiques et vont avoir du mal à revenir au niveau des autres pays de l'Est et encore plus de l'Ouest, même si les causes sont différentes : explosion d'une Fédération construite sous la contrainte, pour la Yougoslavie ; État qui n'a pas eu l'aide communiste et qui se retrouve exsangue lorsque le processus de la dislocation à l'Est se met en route. Dans les deux cas, la radiodiffusion internationale ne sert plus à rien, car il n'y a pas de diaspora yougoslave ou de groupe humain suffisamment important pour cela ; désormais on parle serbe, slovène, croate, albanais, mais il n'y a pas d'entité ni d'identité yougoslave. Quant à l'Albanie, elle avait de l'intérêt lorsqu'elle s'appuyait sur un groupe politique puissant, qu'il s'agisse de l'U.R.S.S. ou de la Chine, mais maintenant à quoi peut servir une radio internationale pour un État de 3500000 individus.

L'affrontement Est-Ouest est remplacé par une mondialisation des modes de consommation et non plus des idéologies. La radiodiffusion 
ne va plus défendre des idées mais des identités et des communautés. Les États qui furent sous la tutelle de l'U.R.S.S. défendent maintenant une culture, une identité propre, non pas en direction de leurs émigrés mais en direction de ceux qui leur sont ou seront utiles commercialement.

Quant aux républiques de l'U.R.S.S. qui se retrouvent dans la CEI, il est curieux de constater que les républiques d'Asie centrale ne cherchent pas à avoir un service extérieur de radiodiffusion et s'appuient toujours sur Moscou pour leur information.

Les républiques du Caucase ont des attitudes différentes. Tout d'abord une volonté d' autonomie par rapport à Moscou, qu'il s'agisse de la Géorgie (qui abandonne la radiodiffusion internationale à l'automne 2005), de l'Arménie, sans parler de la Tchétchénie. Ici la radiodiffusion ne semble être la préoccupation que de l'Arménie, à cause de la diaspora arménienne, sur laquelle compte financièrement l'Arménie. Encore que l'on n'entende plus la Voix de l'Arménie depuis octobre 2006

Les autres États comme la Biélorussie, la Moldavie, l'Ukraine ne semblent pas pressées de développer un service extérieur de radiodiffusion et les raisons semblent être économiques.

Certains Etats n'ont plus de radio internationale ou encore n'émettent-elles que dans la langue de l'émetteur : Norvège, Danemark, Suède, Finlande, Hollande, Slovaquie, Malte, Autriche, etc.

Les anciens émetteurs ont changé parfois de nom et surtout de discours : Radio Moscou s'appelle désormais La Voix de la Russie et son journal est axé uniquement sur l'agenda de M. Poutine, suivi de reportages très conventionnels sur la pêche de l'esturgeon, la population des ours en Sibérie.

En revanche Voice Of America continue de donner des nouvelles en provenance des États-Unis mais aussi donne le point de vue du gouvernement américain sur le terrorisme, la condition féminine dans le monde arabe, et autres sujets polémiques.

Radio Liberty et Radio Free Europe ont dû se reconvertir et quitter le ton anti-communiste du passé. Tout d'abord elles ont quitté Munich pour s'installer à Prague (signe très symbolique). Les contenus se sont modifiés, les budgets ont été réduits et le ton est devenu plus consensuel. 
La montée en puissance des radios internationales religieuses. Le nombre s'est considérablement élevé en dix ans. Un recensement en 2006 nous indique qu'il y a 34 radios de ce type rien qu' aux États-Unis, dont certaines son plus puissantes que les plus classiques des radios internationales comme par exemple : Gospel for Asia en 56 langues ou encore Trans World Radio India qui émet à partir de l'Inde en 63 langues.

De nombreux pays ont des radios religieuses à vocation internationale sur leur territoire ou encore servent de relais ou de bases d'émission d'immenses réseaux comme ceux de Trans World Radio ou Adventist World Radio.

Malgré les changements survenus sur la scène internationale, les contenus des émissions sont toujours aussi insipides et les émissions se déroulent selon un schéma traditionnel :

- journal de quelques minutes sur le pays émetteurs ;

- informations sur l'étranger mais si elles concernent le pays émetteurs ou le pays récepteur, encore est-ce la vision de l'émetteur ;

- selon les stations un magazine qui joue à fond sur la fibre culturelle nationale ou le tourisme national. C'est en gros : voilà qui nous sommes. Que ce soit intéressant ou pas ne semble pas avoir beaucoup d'importance.

\section{Constat global}

La fin de siècle offre un avenir qui apparaît plus simple, plus facile, mais aussi plus risqué car pour la $1^{\text {ère }}$ fois, les États-Unis et des pays alliés vont porter le conflit en Orient, en Irak sans qu'une opposition ne se manifeste réellement. Avec deux blocs, le monde avait un équilibre, même s'il était fondé sur une opposition ; la dislocation de l'Union soviétique laisse les États-Unis seule puissance mondiale et l'équilibre est rompu. La radiodiffusion va elle aussi changer d'interlocuteurs.

\section{Quelques hypothèses}

- La disparition des ondes courtes.

- Les changements politiques (fin d'un monde bipolaire) et techniques doivent avoir des conséquences sur les pratiques et les contenus de la radio internationale. 
- La télévision en continu ne tend-elle pas à remplacer la radiodiffusion internationale.

- La prise de possession des ondes par des groupes ou des communautés en lieu et place des radiodiffuseurs habituels.

\section{Scénarios possibles}

Les ondes courtes si elles franchissent les frontières et les obstacles sont condamnées car les progrès techniques les rendent obsolètes. Les États ne semblent pas prêts à investir pour une onde numérique. Les journaux spécialisés mettent en avant cette innovation technique, mais on n'en voit pas la réalité. De plus il y a un coût économique important et la question semble bien être : faut-il autant investir pour un résultat qui n'est pas aussi essentiel qu'il y parait ? En effet, la technique satellitaire semble bien plus intéressante : World Radio Network qui regroupe actuellement des radiodiffuseurs en français et dans d'autres langues et qui transmet via Internet est offert depuis fin novembre 2002 dans le bouquet Canal Satellite.

Bien évidemment ceci va avoir des effets que nous ne pouvons pas encore mesurer, mais pour lesquels nous pouvons formuler quelques hypothèses. Le fossé va peut-être encore plus se creuser entre ceux qui utilisent l'onde courte et qui, financièrement ne peuvent totalement changer de mode d'émission. En même temps, l'offre de World Radio Network va peut-être permettre à certaines radios de profiter de l'émission satellitaire, mais alors il faudra que les auditeurs possèdent un téléviseur pour écouter la radiodiffusion, si c'est le cas, cet auditeur préfèrera regarder une télévision transnationale, communautaire et à terme thématique, pour s'informer.

Les radios internationales ne semblent plus avoir une grande utilité sauf en cas de conflit, à cause de la simplicité relative d'emploi des ondes courtes et de la facilité pour un petit récepteur autonome de les capter. Mais alors la radiodiffusion deviendra le média d'urgence des radiodiffuseurs internationaux, et des grands radiodiffuseurs uniquement, et seules les grandes nations pourront s'exprimer et donner leur avis, sans tenir compte des particularismes locaux...

La radiodiffusion internationale semble être un moyen d'information qui a ses propres caractéristiques et surtout des spécificités que l'on peut mettre désormais en évidence : 
- c'est le dernier moyen de propagande pour les États ;

- si son objectif premier avoué a été de maintenir le contact avec ses ressortissants, sur un plan national, son objectif désormais est de maintenir le contact avec une communauté, qui est selon les cas : linguistique, religieuse, politique, associative, voire, pourquoi pas, économique ; nous pouvons presque affirmer que la radiodiffusion internationale est devenue, et pour certains États, va devenir, un moyen d'information communautaire, et ce moyen d'information est passé du national au communautaire international. Toutefois, les communautés paraissent actuellement évidentes, et il n'est pas dit qu'avec le temps il ne se forme pas d'autres communautés à faible durée de vie, comme des communautés d'intérêt ayant une cause ou un intérêt particulier à défendre et qui pourraient ainsi devenir des « radios communautaires d'opportunité ».

Et ceci bien que la radiodiffusion internationale ne semble pas devoir être touchée par la commercialisation et ses effets ainsi que le dénonce P. Champagne ${ }^{1}$. Selon J. Mouchon, ${ }^{2}$ plusieurs conséquences résulteraient de cette approche marketing : «La première concerne le corps électoral, traité à l'exemple des consommateurs dans la logique marchande. [...] L'hégémonie du marketing politique a une deuxième conséquence liée à la précédente. Elle concerne la montée en puissance d'un nombre réduit de journalistes. »

L'affrontement idéologique Est-Ouest ayant disparu ou presque, il semble que l'on se dirige vers un affrontement inter-communautaire, non pas un choc des civilisations, mais un affrontement culturel (défense d'une identité culturelle, d'un mode de vie d'une société donnée, aussi bien l'occidentale que l'orientale), et caché sous une apparence religieuse ou économique. Le phénomène n'épargne pas non plus l'Afrique et en particulier le Ghana à en croire A.-J. Tudesq ${ }^{3}:$ «En août 1995, une Association des sociétés de radios privées APBC est constituée, comprenant en mai 2000, 26 radios et 5 télévisions ; [...]

1 CHAMPAGNE Patrick, Faire l'opinion, le nouveau jeu politique, Editions de Minuit, Paris, 1990.

2 MOUCHON Jean, La politique sous l'influence des médias, L'Harmattan, Paris, 1998, p. 62.

3 TUDESQ André-Jean, L'Afrique parle, l'Afrique écoute, Karthala, Paris, 2002, p. 56. 
Elle souhaite aussi défendre l'identité culturelle contre l'excès d'imitation des cultures étrangères. $»^{1}$

Il faut avouer que les auditeurs des ondes courtes ne se situent plus dans les pays de "souveraineté », on aurait presque envie de dire dans les pays riches. Donc qui écoute ? Les pays qui n'ont pas les moyens médiatiques nécessaires et suffisants pour s'affirmer et nous assistons à un phénomène à double tranchant : les pays africains, par exemple, écoutent la radiodiffusion des pays que nous avons appelés « souverains » (occidentaux) et cela a des effets que l'on ne peut bien mesurer dans l'immédiat.

T. Vittin, par exemple, nous précise ${ }^{2}$ que : « L'analphabète haoussa du Niger ou du Nigeria écoutera religieusement les émissions de la $\mathrm{BBC}$, de la DW ou de la VOA diffusées dans sa langue et ce pour améliorer son vocabulaire ou comprendre le sens de certains concepts. Précisons que les journalistes-présentateurs africains recrutés par les radios internationales pour les émissions en langues africaines réalisent un travail de traduction d'autant plus louable que des mots tels que « crise », «pont aérien » ou « inflation » n'ont pas d'équivalent dans ces langues. D'où le recours à des métaphores et d'autres formes imagées pour créer un vocabulaire de vulgarisation, fonction que les auditeurs haoussa, par exemple, ont intégrée dans leur démarche cognitive soustendant - entre autres motifs - leur écoute régulière des radios internationales diffusant dans leur langue. »

Mais il faut rappeler aussi que la majorité des émissions sont diffusées en français, anglais et portugais (langues des anciens colonisateurs). Et comme le précise T. Vittin ${ }^{3}$ : «Quelques grandes langues véhiculaires africaines (swahili, haoussa, lingala...) sont utilisées pour des émissions dont le volume reste faible. » La question demeure entière pour les autres langues véhiculaires africaines : certaines ne figurent pas dans les services des grands radiodiffuseurs et cela peut influer à terme sur leur survie. Cette situation n'accorde-t-elle pas une suprématie

1 Association of Private Broadcasting Company, site Internet de Ghana Media Gateway.

2 VITTIN Théodore, L'impact des radios internationales in La mondialisation des médias contre la censure (sous la direction de Tristan Mattelart), de Boeck \& Larcier S.A, INA, Bruxelles, 2002, p. 95.

3 op. cit., p. 85. 
à certaines ethnies grâce à la langue ? Comme l'exprime T. Vittin ${ }^{1}$ : «Les auditoires restent d'une façon générale circonscrits aux couches urbaines (élèves, étudiants, fonctionnaires, cadres, professions libérales, commerçants) ainsi qu'aux autorités et notables dans les campagnes et villes moyennes (comme les instituteurs, médecins ou fonctionnaires). Ces auditeurs africains constituent des leaders d'opinion et forment une minorité influente dans la vie politique, économique et sociale des États africains. »

Il paraît évident aussi qu'il est plus important pour un leader politique africain ou sud-américain de s'exprimer sur une station internationale que nationale pour trouver une légitimité et ceci pour :

- la taille de l'audience,

- l'internationalité de l'audience, donc sa dimension géographique.

Ce que confirme A.-J. Tudesq ${ }^{2}$ : «Dans le contexte actuel de "mondialisation forcée" auquel sont confrontés les États africains, leur dépendance à l'égard des médias occidentaux se renforce de plus en plus. »

Les raisons de cette dépendance des Africains aux médias internationaux tiennent à une triple dépendance : technologique, économique, savoir-faire, et le recours, entre autres, aux radiodiffuseurs internationaux est inévitable.

Ce fait nuit à la souveraineté nationale des états africains, donc à leur identité nationale mais aussi à leur identité culturelle, car à force de présenter les modes de vie occidentaux il y a obligatoirement une influence sur les modes de vie africains, voire accélération de leur disparition.

Toutefois, il y a un paradoxe que nous pouvons mettre en évidence en relisant le même auteur qui écrivait déjà en $1984^{3}$ : «Si l'on prend l'exemple de la radio en Afrique Noire, la langue utilisée fut d'abord l'anglais ou le français ; la diversité des langues africaines amena souvent à maintenir comme langue officielle le français ou l'anglais ; l'utilisation des langues africaines à la radio est un problème le plus souvent

1 op. cit., p. 85.

2 TUDESQ André-Jean, « Nouvelles technologies de la communication et dépendance renforcée de l'Afrique noire », Mondes en développement, $\mathrm{n}^{\circ} 73,1991, \mathrm{p} .83$.

3 TUDESQ André-Jean, «Les conditions de production et d'écoute. Leurs incidences sur le discours radiophonique », in Aspects du discours radiophonique, sous la direction de Patrick Charaudeau, Dunod, Paris, 1984, p. 14-15. 
politique au moins autant que culturel. La traduction des informations en langue africaine, par exemple en Haute-Volta (Burkina-Faso depuis le 4 août 1984), entraîne un appauvrissement du contenu informatif : ce qui établit une disparité accentuée entre villes et campagnes, entre les cadres dirigeants et la masse de la population. Même dans les quelques pays d'Afrique Noire, qui jouissent d'une homogénéité linguistique, par exemple, la Tanzanie avec l'usage du swahili, des différences apparaissent entre la langue parlée à la radio et celle des paysans. »1

Cet exemple et son ancienneté montrent que la radiodiffusion internationale est encore loin d'une réelle radiodiffusion mondialisée.

Ces phénomènes ne sont pas propres seulement à l'Afrique, on les observe même dans un état comme le Canada sur un autre terrain : «Le marché s'est manifesté dans la radiodiffusion canadienne par le biais des dirigeants d'entreprises «privées »... c'est un lieu commun de l'histoire du Canada que de dire du marché qu'il est continental plutôt que national et que son développement incontrôlé a été perçu comme contraire à l'existence du Canada en tant qu'entité nationale », et plus loin «L'histoire entière du Canada a été celle d'un conflit entre le public, qui est canadien, et le marché, qui a été d'abord américain et, au sens premier du mot, anti-culturel. $»^{2}$ Pour résumer, il y aurait un public canadien (national) dans un marché américain (international).

La communication se mondialise, s'internationalise, de nombreux auteurs l'affirment et nous pouvons nous-mêmes le constater en tant qu'auditeur et/ou téléspectateur. Nous pensons, après écoute des différents journaux parlés, que les radiodiffuseurs de type « communautaires » vont se développer, se renforcer, comme semblent le faire les communautés elles-mêmes. Cette situation ne risque-t-elle pas de provoquer un phénomène de radicalisation de ces communautés et des radiodiffuseurs correspondants, afin de sauvegarder cette identité communautaire ainsi redéfinie?

1 A ce sujet, A.-J. Tudesq signale que dans ce pays le texte des émissions était rédigé en français et diffusé en mooré et dioula, d'où des erreurs de traduction, voire la transmission de fausses informations.

2 THOMAS, A. M., " Audience, Market and Public - An Evaluation of Canadian Broadcasting », Canadian Communications, 1, 1, 1960 : p. 22-24. Cité par Marc RABOY in Accusé de réception (sous la direction de Serge PROULX), L'Harmattan, Champs Visuels, Paris, 1998, pp.108-109. 
Que va-t-il se passer pour les États à faible potentiel en radiodiffusion internationale, ce qui représente presque deux continents : Amérique centrale et du sud et Afrique ? Nous pouvons faire un premier constat : si ces états se passent de radiodiffusion internationale, nous retrouvons les risques déjà mentionnés plus haut, mais dans un cadre de mondialisation de la communication, c'est un fossé qui va séparer ces états des autres. Car là où il y a absence de radiodiffusion internationale, il y a retard d'équipement multimédia ${ }^{1}$. Deuxième constat, les pays en question ne font pas ou peu partie des circuits d'information et il y a un risque d'absence d'image ou au contraire présence de stéréotypes.

La puissance des télévisions internationales va-t-elle suppléer à l'absence de moyens d'information nationaux et internationaux ? Nous constatons ce fait en Afrique du Nord par exemple. L. Madani dans un chapitre concernant la télévision en Algérie $^{2}$, intitule un paragraphe : "L'hégémonie culturelle ininterrompue » et il explicite son propos : «Les modèles culturels européens n'ont pas cessé depuis l'indépendance de s'affirmer comme des vecteurs de savoir et de loisir, d'autant qu'ont été reconduits pour l'essentiel les anciens systèmes de diffusion culturelle. » L. Madani n'oublie pas non plus la radiodiffusion dans son exposé : «Les ondes radiophoniques ont constitué également un lieu non négligeable d'ancrage des nouvelles identités en construction. ». ${ }^{3}$ Ce qui se confirme par deux explications essentielles :

- «D'autant que la dégradation des formes d'expression de la culture traditionnelle qui ne peut trouver de substitut dans une politique culturelle indigente, le taux relativement élevé d'analphabétisme et la prédominance de la communication orale dans la société. $»^{4}$

- «Alors que l'arabe dialectal et le berbère sont les deux langues de communication des larges couches populaires, c'est l'arabe classique qui est imposé comme langue véhiculaire quasi exclusive des programmes nationaux. $»^{5}$

1 Cf. Le rapport mondial sur la communication \& l'informattion 1999-2000 de l'Unesco.

2 MADANI Lofti, «L'antenne parabolique en Algérie, entre domination et résistance », in La mondialisation des médias contre la censure, sous la direction de Tristan Mattelart, de Boeck, Bruxelles, 2002, p. 178.

3 Op. cit., p. 179.

4 Op. cit., p. 180.

5 op. cit., p. 181. 
Nous retrouvons un phénomène semblable en Tunisie où la station nationale RTT (Radio Télévision Tunisienne) essaye péniblement de lutter avec France 2 et la RAI et plus récemment avec des chaînes arabes Al Jazeera et Al Mustakillah. Riadh Ferjani ${ }^{1}$ précise que : " Certaines estimations font état d'une croissance exponentielle du parc d'antennes paraboliques : celui-ci serait passé de 50000 en 1994 à plus de 500000 en 2000. $»^{2}$

Les radios «d'opportunité » continueront de se créer et de disparaître au gré des... opportunités : conflit, entrée dans une communauté économique. La disparition se fait dans le sens de l'abandon d'un service de langue en particulier, mais pas de la station proprement dite. Encore que l'on puisse se demander à quoi peut être utile une radio qui n'émet que dans sa langue nationale pour une communauté très réduite, comme la Finlande.

Nous sommes arrivés au terme de cette recherche et nous devons reconnaître que le bilan global n'est pas en faveur de la radiodiffusion internationale. En dehors des problèmes qu'elle rencontre par rapport à un positionnement dans un système de communication mondiale, ce type de média semble obsolète. Les États ne pourront pas maintenir un mode d'information qui, mis à part les grands, relève plus du bricolage, du discours rapporté (dont l'actualité est discutable) par rapport à l'immédiateté des technologies plus rapides (Internet par exemple) et la réalité des images télévisuelles. Si nous trouvons en Grande-Bretagne, en France, aux États-Unis une réelle volonté d'informer, de nombreux radiodiffuseurs ont assez peu de choses à nous dire, sinon à présenter ou défendre une identité nationale et culturelle ou encore communautaire Ces derniers sont caractérisés par un amateurisme, sympathique certes, mais qui a peu de chances d'avoir un avenir radieux.

D'autre part, même si les citoyens d'un État à parti unique pâtissent d'une sous-information, l'époque des « murs » (de Chine, de Berlin) et

1 FERJANI Riadh, «Internationalisation du champ télévisuel en Tunisie », in $L a$ mondialisation des médias contre la censure, sous la direction de Tristan Mattelart, de Boeck, Bruxelles, 2002, p. 171.

2 Selon, respectivement, une enquête de Radio France internationale citée par Le Figaro, 10 janvier 1995, et une estimation donnée par le cinéaste tunisien Nouri Bouzid aux Cahiers du cinéma, n 557, mai 2001. 
des « rideaux » (de fer ou encore de bambou) est révolue, car il est pratiquement impossible de « rendre sourde » toute une population. Même la Chine est devenue membre en règle de l'OMC depuis le 11 décembre 2001.

Cette « communication-monde » selon A. Mattelart ${ }^{1}$ semble irréversible, car ce sera une communication non-étatisée, mondialisée ou globalisée selon le terme anglo-saxon, comme l'exprime G. Brunel ${ }^{2}$ : « La globalisation actuelle est basée sur le libéralisme économique. Historiquement, celui-ci s'est inspiré des théories de John Locke, d'Adam Smith, de David Ricardo, de Jean-Baptiste Say, de Thomas Robert Malthus et de Léon Walras. Ses opposants se sont regroupés autour de John Stuart Mills, de Karl Marx et de John Maynard Keynes. Signalons que le néolibéralisme actuel a bénéficié des travaux de plusieurs idéologues depuis les années 1930 dont ceux de Friedrich von Hayek et de Milton Friedman. Le moteur actuel de la globalisation est constitué par la stabilité politique et militaire. Présenté comme irréversible, ce changement s'opère souvent par la privatisation de sociétés d'Etat, la déréglementation et la régionalisation des marchés (Marché Commun, ALENA, ZLEA). »

Toujours selon le même auteur : «L'homogénéité engendrée par la globalisation, incomplète actuellement, serait en effet « un rouleau compresseur historique » qui pourrait entraîner l'uniformisation des cultures nationales et régionales, de Londres à Bombay en passant par Hong-Kong et Melbourne. »

En revanche, face à cette globalisation homogène, il y a les tenants d'une vision hétérogène défendue par les théoriciens de l'interculturalisme tels Saïd, Bhabha, Stuart Hall, Clifford, Marcuse et Castells qui supposent que les pays, les régions, les cultures et les sous-cultures ont la capacité de se défendre et ce, d'une manière active. Le récepteur devenant actif.

Il faut remarquer que l'opposition à cette globalisation s'organise, bien qu'elle se fasse encore sous une forme relativement anarchiste (ce qui est préférable à un mouvement unifié qui pourrait être infiltré).

1 MATTELART Armand, La communication-monde, Histoire des idées et des stratégies, La Découverte/textes à l'appui, Paris, 1992.

2 BRUNEL Gilles, "La coopération économique internationale au tournant du millénaire », in La communication internationale, sous la direction de Gilles Brunel et Claude-Yves Charron, Gaëtan Morin éditeur, Boucherville (Québec), Canada, 2002, p. 52. 
Et puis, les derniers sondages de l'écoute radiophonique pour le mois d'octobre 2002 nous indiquent que la station qui a le plus d'audience en France en termes d'écoute cumulée est une station «musicale », c'est une première, et ce n'est plus une station « d'information ». Les goûts et les sujets d'intérêt de la population changent et dans ce contexte, national et international, la situation de la radiodiffusion internationale nous apparait bien fragile et son avenir relativement compromis.

En 1990, Z. R. Nouma concluait ainsi sa thèse sur RFI ${ }^{1}$ : « En tout cas, les bouleversements politiques intervenus récemment dans les pays d'Europe de l'Est, les soubresauts politiques que connaît le continent africain, etc., incitent et doivent inciter à réfléchir à ce que doit être la radio internationale des années 90 et au-delà ». Il ne semble pas que la réflexion ait bien évolué depuis, comme si l'absence de lutte idéologique laissait les adversaires d'hier sans voix, du moins sans message.

Nous terminerons sur cette note à la fois pessimiste et en même temps optimiste. Dans les Cahiers d'Histoire de la Radiodiffusion, nous avons noté à la date du 2 avril 1931 : « Le Petit Radio » reproduit une déclaration désabusée de l'Américain Lee de Forest, inventeur en 1906 de la lampe à trois électrodes. On y lit : «Être considéré comme le «père de la radio » était un honneur dont j'étais fier. Aujourd'hui, je suis dégoûté et honteux de ce titre. Quand nous voyons la vulgarité, la stupidité de la pacotille dont les firmes publicitaires et les stations d'émission emplissent l'éther, je suis sûr que l'on peut me ranger parmi les ennemis publics de l'Amérique $»^{2}$.

Pour notre part nous pensons que Lee De Forest était pessimiste car il assistait dans son pays au triomphe de la radio commerciale de divertissement et qu'il ne fut pas le seul à faire ce constat vis-à-vis des médias, il faut penser à Adorno face au cinéma américain ou Karl Popper face à la télévision. Optimiste quand même, car De Forest est mort en 1961 et il n'a pas assisté à la fin de ce monde bipolaire et de l'importance qu'a prise la radiodiffusion dans ce processus. Et puis il a négligé la dimension humaine de la radiodiffusion internationale, qui relie des hommes quelle que soit leur identité et qui est peut-être encore le dernier média pas trop marchandisé.

1 NOUMA Zanasoumo Roger, Radio France Internationale : instrument de la présence française dans le monde, Thèse pour le Doctorat en Science politique, Université de Lille II, novembre 1990, p. 784.

2 Cahiers d'Histoire de la Radiodiffusion, $\mathrm{n}^{\circ}$ 72, avril-juin 2002. 
Nous pouvons aborder cet avenir sous forme d'hypothèses :

- l'augmentation des audiences : point qui pourrait apporter une information importante mais qui n'a qu'assez peu d'importance, dans le sens où les radiodiffuseurs n'ont pas de moyens scientifiques de mesurer cette audience mais seulement des approches qualitatives à partir de courriers lecteurs et d'estimations empiriques.

- les améliorations techniques qui semblent envisageables à partir du numérique. 


\section{Quelques repères}

\section{Naissance de la radiodiffusion internationale}

La radiodiffusion internationale est née autour des années 1930. Citons les principaux :

- Hollande : 1928

- URSS, Autriche, Allemagne, Kenya : 1929

- Italie : 1930

- Australie, Thaïlande, Vatican et France : 1931

- Grande-Bretagne et Espagne : 1932

- Belgique : 1934

- Suisse, Japon, Chine : 1935

Les États-Unis, pionnier de la radiodiffusion, n'interviendront qu'en 1942, avec Voice of America, c'est-à-dire à cause et pendant la Deuxième guerre mondiale.

La mise en service de la radiodiffusion internationale est dûe à plusieurs raisons essentielles :

- les nations qui possèdent des colonies se doivent d'informer leurs ressortissants nationaux à l'étranger de ce qui se passe en métropole ;

- la montée en puissance des pays fascistes qui mettent en œuvre leur propagande vers l'extérieur, en particulier les colonies aux mains des démocraties et, bien sûr, les pays coloniaux ;

- l'utilisation des ondes courtes qui vont faciliter le développement de la radiodiffusion internationale. Longtemps délaissées, ces longueurs d'ondes inférieures à 100 mètres, permirent tout d'abord, à partir de juin1928, le développement des premières liaisons radiotéléphoniques comme New York - Londres. Au début des années 1950, les ondes courtes restaient le seul moyen de téléphonie transcontinentale aux Etats-Unis.

\section{Bref historique de la radiodiffusion internationale.}

La radiodiffusion internationale a connu trois périodes bien caractéristiques : 1930-1939 : la montée en puissance du média sur fond de propagande 1939-1945 : la propagande de guerre

1945-1989 : la guerre froide

1989 - à nos jours : le nouveau panorama de la radiodiffusion internationale.

\section{La radiodiffusion internationale aujourd'hui.}

Après la guerre froide que constatons-nous :

— des changements techniques : 
- disparition lente mais inéluctable des ondes courtes qui, malgré leur mauvaise qualité de réception parfois, faisaient partie de la radiodiffusion internationale ;

- l'introduction du numérique ;

- utilisations d'internet : on peut écouter toutes (ou presque) les radios internationales sur son ordinateur ;

- utilisation du satellite ;

- utilisation de la FM ;

- introduction du podcast qui permet une écoute différée des émissions de la radiodiffusion internationale ;

- des changements politiques : en l'absence d'un affrontement est-ouest sur fond de propagande a succédé un monde radiophonique plus éclaté du à l'arrivée de nouveaux pays émetteurs (suite à l'effondrement du mur de Berlin) et à la disparition ou à l'affaiblissement d'anciens pays émetteurs qui n'ont plus leur place dans le contexte actuel. D'où une redistribution dans le paysage radiophonique international.

- des changements socio-professionnels car les changements techniques vont modifier 1 'utilisation du média radiophonique.

\section{Quel avenir pour la radiodiffusion internationale?}

Nous pouvons envisager cet avenir à partir de l'émission, des contenus, de la réception et de la technique.

Une réponse immédiate n'accorderait pas un grand avenir pour la radiodiffusion internationale : disparition de certains pays sur la scène radiophonique internationale ; en effet depuis la fin de la guerre froide, donc depuis 1945, les pays suivants ont disparu des ondes internationales :
- Hollande ;
- Suède,
- Danemark ;
- Norvège ;
- Finlande ;
- Slovaquie ;
- Arménie ;
- Autriche ;

En revanche de nouveaux acteurs sont arrivés :

- Moldavie ;

- Estonie ;

- Lettonie,

- Lituanie 


\section{À partir de l'Alaska : \\ KLNS en 3 langues : mandarin, russe, anglais,}

Importance prise par les radiodiffuseurs religieux

\section{À partir de l'Afrique du Sud :}

Trans World Radio Africa en 49 langues

À partir de l'Allemagne :

Evanguliums-Rundfunk en 10 langues : allemand, arabe, croate, espagnol, farsi, français, grec, russe, sorani, turc.

Freie Volkmission en allemand et en anglais.

Lutherische Stunde en allemand

Missionswerke Arche en allemand

Missionswerke Heukelbach en allemand

Radio Santec en 6 langues : allemand, anglais, espagnol, français, polonais, portugais,

À partir de l'Australie :

CVC International en 4 langues : anglais, indonésien, mandarin, hindi (par la Voix de l'Asie)

HCJB World Radio Australia en 15 langues.

À partir de l'Autriche :

Trans World Radio en 47 langues

À partir du Canada

Eglise du Christ en français

À partir du Chili

Voz cristiana en portugais et en espagnol

\section{À partir du Costa Rica}

The Caribbean Beacon (University network relay)

À partir de Chypre :

Radio Ibrahim en arabe
À partir de l'Equateur :

HCJB (La Voix des Andes) en 18 langues.

à partir des Etats-Unis :

Adventist World Radio (AWR) en 35 langues.

BYU Radio en anglais

Christian Science Sentinel en allemand, anglais et russe.

Eternal Good News en anglais

Far East Broadcasting Radio Company, Inc

Gospel for Asia en 56 langues.

HCJB World Radio

High Adventure Ministries

Kaij International

KIMF

KJES en anglais et en espagnol

KTBN Shortwave Radio

KVOH - La Voz de Restauracion en espagnol

Leading the way en farsi et en russe

Pan American Broadcasting en anglais, arabe, farsi.

Radio Payam-E Doost en farsi

The overcomer ministry en anglais

The University Network (Dr Gene Scott) en anglais

Trans World Brodcasting Ministry en mandarin

Trans World Radio

Transformation Media International (SW Station Project)

Truth for the world en mandarin

WBOH/WTJC - Fundamental Brodcasting Network (FBN) en anglais

WEWN - Global Catholic Network (ETWN) en anglais, en espagnol.

WHRA (World Harvest Radio) en anglais

WHRI (World Harvest Radio) en anglais, arabe, espagnol

WINB en anglais 


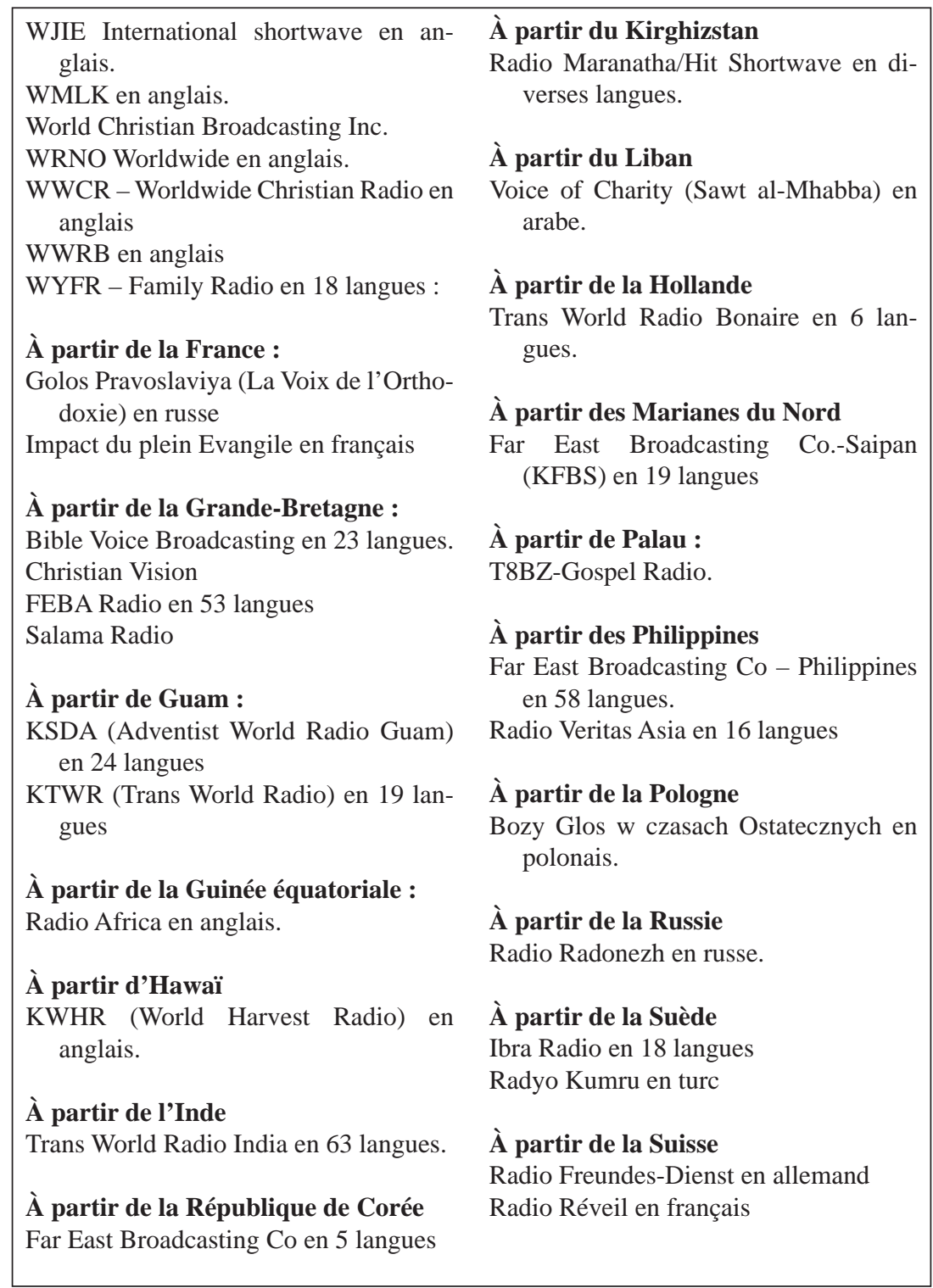


